\title{
Stray dogs and cats as potential sources of soil contamination with zoonotic parasites
}

\author{
Katarzyna Szwabe', Joanna Błaszkowska² \\ ${ }^{1}$ Department of Biology and Medical Parasitology, Medical University of Lodz, Poland \\ 2 Department of Diagnostics and Treatment of Parasitic Diseases and Mycoses, Medical University of Lodz, Poland
}

Szwabe K, Błaszkowska J. Stray dogs and cats as potential sources of soil contamination with zoonotic parasites. Ann Agric Environ Med. 2017; 24(1): 39-43. doi: 10.5604/12321966.1234003

\section{Abstract}

Introduction and objective. The main source of many zoonoses is soil contaminated with feline and canine faeces. Thus, the aim of this study was to estimate the prevalence of intestinal parasites in stray dogs and cats adopted in Lodz shelter (Poland).

Materials and methods. In total, 163 faecal samples were collected from 95 dogs and 68 cats from 2011 to 2012 . The samples were processed by sedimentation techniques using Mini Parasep ${ }^{\circledR}$ SF.

Results. Six parasite genera belonging to protozoa, cestoda, and nematoda, were found in dogs, while eight were found in cats. Out of the $163 \mathrm{fecal}$ samples, 37.4\% were positive for the presence at least one species of intestinal parasites. The majority of positive dog samples contained eggs from Toxocara and Trichuris genera, and the family Ancylostomatidae, while Toxocara and Taenia eggs, as well as Cystoisospora oocysts, predominated in cat faeces. A significantly higher prevalence of parasites was noted in cats $(48.5 \%)$ than in dogs $(29.5 \%)\left(x^{2}=6.15, P=0.013\right)$. The Toxocara genus was the most prevalent parasite in both populations; eggs were found in $27.9 \%$ and $16.8 \%$ of cats and dogs, respectively. Animals younger than 12 months of age showed higher infection rates with Toxocara, but differences were not statistically significant. The average numbers of Toxocara eggs/gram of faeces in positive puppy and kitten samples were over 5 and 7 times higher than in older dogs and cats, respectively. Mixed infection were found in dogs (5.3\%) and cats (8.8\%).

Conclusions. Cat faeces represent a more important potential source of environmental contamination with zoonotic parasites than dog faeces. Among the detected parasites of stray dogs and cats, Toxocara present an important zoonotic risk for the local human population, especially children.

\section{Key words}

zoonoses, stray dogs/cats, public health, parasite eggs, Toxocara spp., gastrointestinal parasites

\section{INTRODUCTION}

The growing number of companion animals, including cats and dogs, poses serious public health, veterinary and socioeconomic problems worldwide. According to IFAHEurope (the International Federation for Animal Health Europe), there are 223 million dogs and 220 million cats throughout the world, excluding stray animals. In Europe, the largest pet dog populations are in France, amounting to 8.8 million, while both Italy and Poland each have over 7.5 million. Currently, the global dog population (pet, roaming, stray, and feral animals) is estimated to be more than 500 million [1].

Animals are the source of many pathogens: dogs themselves are associated with more than 60 zoonotic diseases, most of which can be dangerous for human health [2]. Of all the parasites of dogs and cats, the gastrointestinal species are regarded as the most important from an epidemiological point of view. The most frequent intestinal parasites of these animals belong to genera from different taxonomic groups, especially protozoa and helminths: Giardia, Cystoisospora, Taenia, Dipylidium, Echinococcus, Toxocara, Toxascaris, Ancylostoma, Uncinaria, Capillaria, Trichuris $[3,4,5,6]$. Most of them can infect humans, but Toxocara, Ancylostoma and Echinococcus have the greatest epidemiological importance

Addres for correspondence: Katarzyna Szwabe, Department of Laboratory Diagnostics and Clinical Biochemistry, Central Clinical Hospital of Medical University in Łódź, ul. Pomorska 251, 92-213 Łódź

E-mail: katarzyna.szwabe@umed.lodz.pl

Received: 18 November 2013; accepted: 5 March 2014; first published on February 2017
$[7,8]$. Their dispersion forms (oocysts, cysts, eggs, larvae) are deposited in the environment by the faecal route. It has been estimated that a population of 100,000 dogs deposits between 3-11 tons of faeces daily into the environment [9]. Humans can become accidental hosts through contact with infective eggs and (oo)cysts from contaminated soil, unwashed raw vegetables, or by direct contact with infected animals [7, 10]. However, contact with soil is still believed to be the most important route for human infection. Most commonly, children are infected after exposure to contaminated soil while playing in sandpits or playgrounds. For this reason, many studies have been carried out in recent years to determine the prevalence of geohelminth eggs in the recreational areas [11, 12]. Parasite control programmes should be based on active monitoring of parasite distributions and identification of specific risk factors [13].

The growing numbers of abandoned animals have also become a problem in many European countries as the constant number of shelters, together with the increasing number of abandoned animals, means that not all strays can be accepted. According to a report by the Chief Veterinary Inspector of Poland, at the end of 2011, the respective stray dog and cat populations of 150 shelters were estimated to number 100,265-20,470. Voslarova and Passantino [14] note that the number of dogs which arrived at shelters in Italy in 2009 was 95,648 , although the estimated population of stray dogs was 10 times greater. It is estimated that in Italy, about $25 \%$ of all pets are abandoned every year.

Stray or abandoned dogs and cats adopted into care shelters are often in poor health with various parasitic diseases, and 
they are viewed as the most important source of zoonoses. It was noted that environmental contamination with helminth eggs or protozoa (oo)cysts is correlated with the prevalence of intestinal parasite infections in animal populations [7].

\section{OBJECTIVE}

The aim of this study was to estimate the prevalence of intestinal parasites in stray dogs and cats adopted in a shelter in Lodz, Poland, as a means of estimating the zoonotic risk associated with these infections for local human population. This knowledge of the spectrum of animal parasites and their epidemiology is important in the formulation of effective zoonotic disease control measures.

\section{MASTERIALS AND METHOD}

Study area. The study was carried out in urban area in the city of Lodz in Poland, a city situated in a temperate climatic zone with four distinct seasons. Lodz is the third-largest city in Poland located in the central part of the country, with a population estimated at about 737,100 residents. According to estimates available at the public health service, the pet dog population of Lodz exceeds 70,000, and almost 700 dogs and 100 cats are resident in one shelter located in the city area.

Sample collections. Research was carried out during 20112012 on 95 dogs and 68 cats adopted by the Animal Shelter located in the city of Lodz. All newly-adopted animals were subjected to mandatory quarantine. During the first defecation of the animals, excrement was collected by the workers at the shelter. The whole stool was placed into a disposable plastic container with $5 \%$ formalin for fixation and stored at about $4{ }^{\circ} \mathrm{C}$ for parasitological examination. The individual data of the animals such as age, gender and date of collection was recorded for each sample. The collected material was delivered twice a week from the shelters to the Department of Diagnostics and Treatment of Parasitic Diseases and Mycoses at the Medical University in Lodz.

Laboratory procedure. All animal faeces were initially examined macroscopically for the presence of tapeworm proglottids or mature worms. From each stool, one sample weighing about $0.5 \mathrm{~g}$ was examined for developmental forms of intestinal protozoa and helminths using a Mini Parasep ${ }^{\oplus}$ SF faecal parasite concentrator (Diasys Europe Ltd.). This system, consisting of a mixing and a centrifuge tube, rapidly eliminates undigested elements and fat from examined materials and concentrated samples. Concentration takes place through a four-step process involving sample preparation, emulsification, centrifugation and examination.

The spoon on the end of the filter was used to introduce the faecal samples into a mixing tube containing a mixture of reagents: $2.4 \mathrm{ml}$ of $10 \%$ buffered formalin, $0.9 \mathrm{ml}$ ethyl acetate and one drop of Triton X-100. Next, the Parasep was immediately sealed by screwing the filtrate/thimble sedimentation cone onto the mixing chamber and the mixture was shaken for 15-20 seconds to obtain an emulsion. The Parasep was then inverted to allow the mixture tube filtered through the filter thimble and was centrifuged at 3,000 $\mathrm{g}$ for $1 \mathrm{~min}$.
Direct wet smears were prepared from sediment with a drop of sterile water. All the sediment in each sample was examined: 6-7 slides in total. The prepared slides were evaluated using a Nikon ECLIPSE E-200 light microscope at 100x and 400x magnification. The intensity of eggs excreted by individual species of parasite was expressed as mean number of eggs per gram of faeces. Parasite eggs and (oo) cysts were identified based on structural and morphometric features [15]. In formalin-fixed stool samples, the presence of Giardia or Cryptosporidium coproantigens was not examined.

Due to their great morphological similarity, eggs from the genus Capillaria and from the cestode family Taeniidae were not differentiated to species. Similarly, eggs belonging to the Uncinaria or Ancylostoma genera were included into the Ancylostomatidae family.

Statistical analysis. The overall prevalence of intestinal parasites was estimated as the number of animals found to be positive for the presence at least one species of parasite divided by the total number of animals examined. The prevalence of each parasite was calculated as the number of infected individuals over the total number of dogs/cats examined. Comparison between proportions were carried out using the Chi-squared test of Pearson or Fisher's exact test if any expected frequency was lower than 5. The MannWhitney $U$ test was used to compare the average densities of Toxocara eggs found in positive samples. All statistical analyses were performed using STATISTICA v. 10 software. Values of $\mathrm{P}<0.05$ were taken as significant.

\section{RESULTS}

Of the 163 faecal samples, $37.4 \%$ were positive for the presence of at least one species of intestinal parasite. The overall prevalence of parasites in the examined population were found to be $48.5 \%$ for cats and $29.5 \%$ for dogs (Tab. 1), and these values were found to be significantly different $\left(\chi^{2}=6.15\right.$, df. $=1 ; \mathrm{P}=0.013)$. The majority of positive dog samples contained developmental forms of parasites from Toxocara and Trichuris genera, and the Ancylostomatidae family, while genera such as Toxocara, Taenia and Cystoisospora dominated in cat faeces. The most frequently observed parasites, in both the cat and dog populations, was Toxocara.

Table 1. Infections with intestinal parasites in coproscopically examined animals belonging to 2 age groups

\begin{tabular}{lccc}
\hline Animals & Age groups & $\begin{array}{c}\text { No. of examined } \\
\text { stool samples }\end{array}$ & $\begin{array}{c}\text { No. of positive } \\
\text { samples (\%) }\end{array}$ \\
\hline \multirow{2}{*}{ Dogs } & $6-12$ months & 38 & $13(34.2)$ \\
\cline { 2 - 4 } & over 12 months & 57 & $15(26.3)$ \\
\cline { 2 - 4 } Cats & Total & 95 & $28(29.5)$ \\
\cline { 2 - 4 } & $6-12$ months & 24 & $9(37.5)$ \\
\cline { 2 - 4 } & over 12 months & 44 & $24(54.6)$ \\
\hline & Total & 68 & $33(48.5)$
\end{tabular}

A total of 8 species of intestinal parasite were identified microscopically in cat faeces and 6 in dog faeces (Tab 2, 3). A single mature form of $T$. canis was found in 4 fecal samples from young dogs. Additionally, tapeworm proglottids were found in cat stools: Taenia taeniaeformis and Dipylidium 
caninum. Only the cats were infected by tapeworms, especially Taenia taeniaeformis; in addition, Cystoisospora oocysts and Giardia cysts were found only in cat samples.

Due to the fact that the vast majority of animals adopted by the shelter were males, the ratio of males to females being 4:1, no attempt was made to evaluate the prevalence of intestinal parasites according to gender. The animals were divided into 2 groups based on age: a younger group aged over 12 months and an older group of more than one year old. In the examined population, no cat was under 3 months of age and no dog was younger than 6 months.

The overall prevalence of intestinal parasites in the cat population up to 12 months was higher than that seen in the younger group under 12 months (Tab. 2), but the difference was not statistically significant $\left(\chi^{2}=1.81 ; \mathrm{df}=1 ; \mathrm{P}=0.179\right)$. Also, the prevalence of $T$. cati did not differ significantly between the 2 age groups: $10.3 \%$ in young cats ( $<12$ months) and $17.7 \%$ in older animals ( $>12$ months $)\left(\chi^{2}=0.03\right.$; df. $=1$; $\mathrm{P}=0.862$ ). Moreover, the intensity of faecal eggs excreted was 7 times higher in young cats than in older cats (Fig. 1). The average number of eggs/g of faecal samples from animals under the age of 12 months was significantly higher $(\mathrm{P}<0.05)$ than from cats over 12 months old. Great variation was seen between individuals with regard to faecal egg and (oo)cyst excretion; the number of $T$. cati eggs per positive feline sample ranged from 1-906; mean value 249.6 eggs/g, Taenia eggs ranged from 3-143, mean 109.6 eggs/g and Cystoisospora oocysts ranged from 1-43; mean 13.8 oocyts/g.

Table 2. Prevalence (\%) of gastrointestinal parasites relative to age of cats

\begin{tabular}{lccc}
\hline & \multicolumn{3}{c}{ Age groups } \\
\cline { 2 - 4 } Species/genus/family & 6-12 months & over 12 months & Total \\
\cline { 2 - 4 } & \multicolumn{3}{c}{ Prevalence $^{\mathrm{a}}$ (Relative prevalence) } \\
\hline Toxocara cati & $10.3(21.21)$ & $17.7(36.36)$ & $27.9(57.58)$ \\
\hline Capillaria & 0 & $1.5(3.03)$ & $1.5(3.03)$ \\
\hline Ancylostomatidae & 0 & $1.5(3.03)$ & $1.5(3.03)$ \\
\hline Taenia & 0 & $8.8(18.8)$ & $8.8(18.8)$ \\
\hline Dipylidium caninum & $1.5(3.03)$ & $2.9(6.06)$ & $4.4(9.09)$ \\
\hline Cystoisospora & $1.5(3.03)$ & $10.3(21.21)$ & $11.8(24.24)$ \\
\hline Spirometra erinaceieuropaei & 0 & $1.5(3.03)$ & $1.5(3.03)$ \\
\hline Giardia & 0 & $2.9(6.06)$ & $2.9(6.06)$
\end{tabular}

a - Prevalence calculated by dividing the number of cats infected with individual parasite by the total number of cats in each age group.

${ }^{b}$ - Percentages calculated as the number possessing an individual parasite divided by the total positive cats.

For dogs, the overall prevalence of parasite infections was similar in the 2 age groups $\left(\chi^{2}=1.1 ; \mathrm{df}=1 ; \mathrm{P}=0.294\right)$. The prevalence of T. canis in the dog population over 12

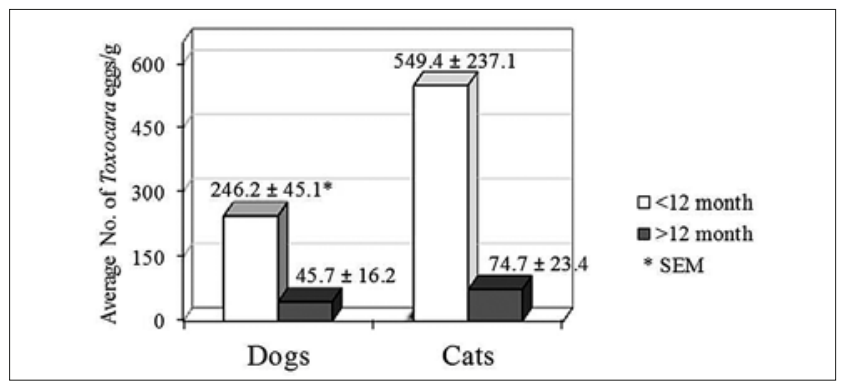

Figure 1. Average density ( \pm SEM) of Toxocara eggs/g in all positive samples
Table 3. Prevalence (\%) of gastrointestinal parasites relative to age of dogs

\begin{tabular}{|c|c|c|c|}
\hline \multirow{3}{*}{ Species/genus/family } & \multicolumn{3}{|c|}{ Age groups } \\
\hline & $6-12$ months & over 12 months & Total \\
\hline & \multicolumn{3}{|c|}{ Prevalence $^{\mathrm{a}}$ (Relative prevalence) ${ }^{\mathrm{b}}$} \\
\hline Toxocara canis & $9.5(32.14)$ & $7.4(25)$ & $16.8(57.14)$ \\
\hline Trichuris vulpis & $5.3(17.86)$ & $3.2(10.7)$ & $8.4(28.57)$ \\
\hline Ancylostomatidae & $1.1(3.57)$ & $6.3(21.43)$ & $7.4(25)$ \\
\hline Capillaria & 0 & $1.1(3.57)$ & $1.1(3.57)$ \\
\hline Cystoisospora & 0 & $1.1(3.57)$ & $1.1(3.57)$ \\
\hline Toxascaris leonina & $1.1(3.57)$ & 0 & $1.1(3.57)$ \\
\hline
\end{tabular}

a - Prevalence calculated by dividing the number of cats infected with individual parasite by the total number of dogs in each age group.

b - Percentages calculated as the number possessing an individual parasite divided by the total positive dogs.

Table 4. Number of animals with single and mixed infections

\begin{tabular}{lcc}
\hline No. of parasites in fecal samples & No. of dogs & No. of cats \\
\hline 0 & 67 & 35 \\
\hline 1 & 23 & 27 \\
\hline 2 & $4^{\mathrm{a}}$ & $4^{\mathrm{b}}$ \\
\hline 3 & $1^{\mathrm{c}}$ & $2^{\mathrm{d}}$
\end{tabular}

a - Ancylostomatidae/Trichuris vulpis (twice); Ancylostomatidae/Toxocara canis (twice)

b - Toxocara cati/Giardia sp.; Toxocara cati/Cystoisospora sp; Toxocara cati/Taenia sp.; Cystoisospora sp. /Giardia sp.

c- Ancylostomatidae/Toxocara canis/Capillaria sp.

d - Toxocara cati/Cystoisospora sp./Taenia taeniaeformis; Ancylostomatidae/Capillaria sp. / Spirometra erinaceieuropaei.

months (7.4\%) was slightly lower than seen in the younger group (9.5\%) (Tab. 3). The number of eggs/gram of faeces in positive samples was found to be over 10 times higher in the puppies than the adult dogs $(\mathrm{P}<0.05)$ (Fig. 1). The dogs were 7 times more likely to be infected with parasites from Ancylostomatidae family than cats, while none of the Taenia type eggs were found in samples from dogs. The eggs of Toxascaris leonine was detected only in one puppy sample. In positive canine samples, the average number of T. canis eggs was found to be $152.5 / \mathrm{g}$, ranging from 1-228, Trichuris vulpis was 25.8 eggs/g, ranging from 1-28, and Ancylostomatidae family was 40.8 eggs/g, ranging from 1-58.

In this study, double and triple infections were observed in both the cat and dog populations (Tab. 4). Ancylostomatidae nematode invasions were associated with T. canis, T. vulpis or Capillaria invasions, while in feline samples, Cystoisospora was associated with the presence of $T$. cati eggs, and/or with Taenia eggs.

\section{DISCUSSION}

The presented study shows that the stray animals adopted by a shelter located in the city of Lodz, Poland, were host to a wide range of intestinal parasites, which includes nematodes, cestodes and protozoans. A significantly higher overall prevalence of parasites was noted in cats (48.5\%) than in dogs (29.5\%). A similar high frequency of parasite infections - 42.2\%, was identified by Ładczuk and Balicka-Ramisz [16] in cats from shelters situated in the area of Szczecin, Poland. Other epidemiological studies have confirmed that stray cat populations are a very important reservoir of protozoa and helminth parasites $[17,18]$. A study conducted in Poland's 
capital city, Warsaw, also indicated that a high percentage of kennel dogs (56.5-80.9 \%) were infected with intestinal nematodes, while only $3.5 \%$ of household animals were positive [19]. The prevalence of intestinal parasites in the stray and refuge dog populations of Europe varies widely, from $25 \%$ up to $63 \%[3,4,5]$.

In this study, the genus Toxocara was found to dominate among parasites detected in faecal samples of cats and dogs. Toxocara spp. eggs were found in $27.9 \%$ of cats and 16.8 $\%$ of dogs. A similar result was reported in Ładczuk and Balicka-Ramisz [16] where the prevalence of T. cat $i$ in refuge cats was found to be $24.21 \%$. However, other studies based on necropsy observed different levels of prevalence of this species in cats ranging from $8-42.6 \%[20,21]$. Also, many studies from Europe report that the most common intestinal parasites occurring in stray and abandoned dogs is T. canis $[4,5,17]$.

Some age-dependent analyses report high levels of parasite infection in the dog and cat populations, especially in animals up to 3 months, and in those $3-6$ months of age $[7,22]$. In particular, T. canis and T. cati are more often recorded in young animals than in adults. Szabova et al. [4] show a considerably higher level of $T$. canis infection present in young dogs (53.2\% of pups up to 6 months) than in the older animals (18.8\% of dogs over one year of age). Rubel at al. [23] report that the prevalence of T. canis is 20 times higher in dogs less than one year old than in those that are older. In the presented study, 9.5\% of puppies and $7.4 \%$ of dogs over one year were found to be infected with T. canis, but this difference was not statistically significant. As also seen in the current study, Awoke et al. [24] found no statistically significant differences between age and the frequency of infection with T. canis in dogs. In the presented study, much higher infection rates with T. cati were observed in cats over 12 months old (17.7\%) than in animals under 12 months old (10.3\%). This could be connected with the predatory lifestyle of stray animals and their consumption of small animals infected with Toxocara, such as small rodents and birds.

Hookworm infections with either Ancylostoma caninum or Uncinaria stenocephala tend to be variable and focused in regional geographic areas. While the dominant species in the temperate climate zone is Toxocara genus, hookworms are the most common intestinal parasite detected in the population of dogs and cats in the tropical and subtropical climate zones. As described by Zewdu et al. [25], 50\% of the necrosed dogs in Ambo, Ethiopia, were found to be positive for A. caninum. According to Chen et al. [26], although A. caninum and $A$. tubaeforme have been documented to be the most common species occurring in the warmer ranges of China, A. caninum is also prevalent in the cold regions of the country. Szabova et al. [4] also demonstrate a high prevalence of nematodes from the Ancylostomatidae family in dog populations from 2 shelters located in the Slovak Republic, the exact values being $25.6 \%$ and $26.8 \%$. In the presented study, the prevalence of hookworms in stray dogs was $7.4 \%$, but eggs were 6 times more frequently found in faecal samples of dogs over one year old, compared with younger dogs up to 12 months old. In the examined population of cats, only one animal over one year was infected with hookworm.

In the presented study, Trichuris vulpis was the third most common species identified in faecal samples of dogs, and its prevalence in canine population was $8.4 \%$. A similar T. vulpis prevalence was noted in dog populations from other European kennels $[4,5,19]$. While no Trichuris genus infection was seen in the examined cat population, only cats were infected by tapeworms (14.7\%), particularly from the Taeniidae family $(8.8 \%)$. However, other authors have demonstrated a less than $3.4 \%$ of Taenia-type eggs detections in faecal samples from dogs living in some temperate zone countries $[4,17]$.

In the current study, some nematode genera, of such as Toxascaris and Capillaria, were detected sporadically both in canine and feline faeces. According to other authors, Capillaria eggs can also occasionally be found in the faeces of these animals $[17,18]$. It should be noted that T. leonina occurs less frequently (1-8\%) than the Toxocara genus in northwest and central Europe $[4,16,19]$, but is highly prevalent in subtropical and tropical regions [27].

Stray dogs and cats are often infected with parasitic protozoa from coccidian families. In this study, only oocysts of Cystoisospora were detected, and stray cats (11.8\%) were more often found to be infected than dogs (1.1\%). Research from the Slovak Republic reports a similar prevalence of Cystoisospora invasion (10.8\%) in the dog population, but other authors have shown that its prevalence in stray and refuge animals varies from $0.8 \%-80 \%[4,16,21]$. Also, various genera of coccidia have been detected in German pets: the prevalence of Cystoisospora being 5.6\% and 6\% for dog and cat populations, respectively [17]. Some parasites occurring in cats and dogs, both protozoa and helminths, are responsible for important zoonotic diseases, including welldocumented diseases such as echinococcosis, toxocarosis, ancylostomatidosis and trichuriasis, as well as emerging and re-emerging infections, such as cryptosporidiosis and giardiasis $[6,8,28]$.

The results of the presented study indicate that the main risks to public health are Toxocara, responsible for the development of larva migrans syndromes and ocular toxocarosis in man. Trichuris, hookworm and Giardia infections in stray animals detected by coprological examination were rare or occasional. The tapeworm eggs from Taeniidae family present in stool samples were not differentiated to the species, hence the presence of Echinococcus granulosus in the examined animals was not confirmed.

The present study also reveals a strong correlation between the gastrointestinal parasitic fauna of the local populations of stray dogs and cats, and the presence of egg helminths detected at public recreation areas in the city of Lodz in Poland, as reported in previous research [12]. Eggs belonging to the same genera were detected both in samples of soil and animal feces. In the above studies, genus Toxocara predominated, hookworm eggs were detected less frequently, while Trichuris eggs were found occasionally.

\section{CONCLUSIONS}

The results of the presented study show that the stray cat population represents a more important potential reservoir of the parasites than the dog population. The homeless cats and dogs living in the city of Lodz infected by various zoonotic intestinal parasites pose a risk of soil contamination with dispersion forms of protozoa and helminths, especially Toxocara eggs.

These results indicate the necessity for a reduction in the numbers of stray animals in this urban area in order to 
minimize risks of zoonotic parasitic infection to humans. Local authorities should implement effective strategies for homeless dog and cat population control, such as the creation of new shelters with adequate veterinary care, large-scale sterilization of animals as well as greater enforcement of laws concerning pet ownership and the educating of owners. But above all, to eliminate the problem of abandoned animals, people should be more responsible and compassionate.

\section{Acknowledgement}

This study was supported by Project No. 502-03/1-01301/502-14-011 at the Medical University in Lodz, Poland.

\section{REFERENCES}

1. Hsu Y, Severinghaus L, Serpell J. Dog keeping in Taiwan: Its contribution to the problem of free-roaming dogs. J of Appl Anim Welf Sci. 2003; 6(1): 1-23.

2. Macpherson CNL. Human behaviour and the epidemiology of parasitic zoonoses. Intl J Parasitol. 2005; 35: 1319-1331.

3. Dubna S, Langrova I, Napravnik J, Jankovska I, Vadlejch J, Pekar S, Fechtner J. The prevalence of intestinal parasites in dogs from Prague, rural areas, and shelters of the Czech Republic. Vet Parasitol. 2007; 145(1-2): 120-128.

4. Szabova E, Juris M, Miterpakova M, Antolova D, Papajova I, Sefcikova $\mathrm{H}$. Prevalence of important zoonotic parasites in dog populations from the Slovak Republic. Helminthologia 2007; 44(4): 170-176.

5. Claerebout E, Casaert S, Dalemans A-C, Wilde DN, Levecke B, Vercruysse J, Geurden T. Giardia and other intestinal parasites in different dog populations in Northern Belgium. Vet Parasitol. 2009; 161: 41-46.

6. Deplazes P, van Knapen F, Schweiger A, Overgaauw PA. Role of pet dogs and cats in the transmission of helminthic zoonoses in Europe, with a focus on echinococcosis and toxocarosis. Vet Parasitol. 2011; 182(1): 41-53.

7. Overgaauw PA, van Knapen F. Veterinary and public health aspects of Toxocara spp. Vet Parasitol. 2013; 193(4): 398-403.

8. Da Silva AM. Human Echinococcosis: A Neglected Disease. Gastroenterol Res Pract. 2010; http://dx.doi.org/10.1155/2010/583297.

9. Beck AM. The Ecology of Stray Dogs: A Study of Free-Ranging Urban Animals. York Press, Baltimore 1973; first ed: 95.

10. Kłapeć T, Borecka A. Contamination of vegetables, fruits and soil with geohelmints eggs on organic farms in Poland. Ann Agric Environ Med. 2012; 19(3): 421-425.

11. Bajor H, Kłapeć T. Contamination of soil with eggs of geohelmints in recreational areas in the Lublin region of Pland. Ann Agric Environ Med. 2012; 19(2): 267-270.
12. Blaszkowska J, Wojcik A, Kurnatowski P, Szwabe K. Geohelminth egg contamination of children's play areas in city of Lodz (Poland). Vet Parasitol. 2013; 192: 228-233.

13. Martinez-Carrasco C, Berriatua E, Garijo M, Martinez J, Alonso FD, de Ybanez RR. Epidemiological study of non-systemic parasitism in dogs in southeast Mediterranean Spain assessed by coprological and postmortem examination. Zoonoses Public Health. 2007; 54(5): 195-203.

14. Voslarova E, Passantino A. Stray dog and cat laws and enforcement in Czech Republic and in Italy. Ann Ist Super Sanita. 2012; 48(1): 97-104.

15. Bowman DD. Georgis' parasitology for veterinarians. $9^{\text {th }}$ ed. Elsevier Urban \&Partner, 2012.

16. Ładczuk D, Balicka-Ramisz A. Occurrence of parasites in the alimentary canal of cats from Szczecin area, Poland. Ann Parasitol. 2010; 56(3): 235-242.

17. Barutzki D, Schaper R. Results of Parasitological Examinations of Faecal Samples from Cats and Dogs in Germany between 2003 and 2010. Parasitol Res. 2011; 109: 45-60.

18. Sowemimo AO. Prevalence and intensity of gastrointestinal parasites of domestic cats in Ode -Irele and Oyo communities, Southwest Nigeria. J Parasitol Vector Biol. 2012; 4(1): 7-13.

19. Borecka A. Prevalence of intestinal nematodes of dogs in the Warsaw area, Poland. Helminthologia. 2005; 42: 35-39.

20. Zibaei M, Sadjjadil SM, Sarkari B. Prevalence of Toxocara cati and other intestinal helminthes in stray cats in Shiraz, Iran. Trop Biomed. 2007; 24(2): 39-43.

21. Esmaeilzadeh M, Shamsfard M, Kazemi A, Khalafi SA, Altome SA. Prevalence of Protozoa and Gastrointestinal Helminthesin Stray Cats in Zanjan Province, North-West of Iran. Iranian J Parasitol. 2009; 3(4): 71-75.

22. Habluetzel A, Traldi G, Ruggieri S, Attili AR, Scuppa P, Marchetti R, Menghini G, Esposito F. An estimation of Toxocara canis prevalence in dogs, environmental egg contamination and risk of human infection in the Marche region of Italy. Vet Parasitol. 2003; 113: 243-252.

23. Rubel D, Zunino G, Santillan G,Wisnivesky C. Epidemiology of Toxocara canis in the dog population from two areas of different socioeconomic status, greater Buenos Aires, Argentina. Vet Parasitol. 2003; 115: 275-286.

24. Awoke E, Bogale B, Chanie M. Intestinal Nematode Parasites of Dogs: Prevalence and Associated Risk Factors. Int J Anim Vet Adv. 2011; 3(5): 374-378.

25. Zewdu E, Semahegn Y, Mekibib B. Prevalence of helminth parasites of dogs and owners awareness about zoonotic parasites in Ambo town, central Ethiopia. Ethiop Vet J. 2010; 14(2): 17-30.

26. Chen J, Xu M-J, Zhou D-H, Song H-Q, Wang Ch-R, Zhu X-Q. Canine and feline parasitic zoonoses in China. Parasit Vectors. 2012; 5: 152. http://www.parasitesandvectors.com/content/5/1/152.

27. Dalimi A, Sattari A, Motamedi G. A study on intestinal helminthes of dogs, foxes and jackals in the western part of Iran. Vet Parasitol. 2006; 142: 129-133.

28. Esch KJ, Petersen CA. Transmission and epidemiology of zoonotic protozoal diseases of companion animals. Clin Microbiol Rev. 2013; 26(1): 58-85. 